OPEN ACCESS

Edited by:

Younes Smani

Institute of Biomedicine of Seville

(IBIS), Spain

Reviewed by:

Oleg Reva,

University of Pretoria, South Africa

Karl Hassan,

The University of Newcastle, Australia

*Correspondence:

Laura Álvarez-Fraga

laura.alvarez.fraga@sergas.es

tORCID:

Kelly Conde-Pérez

orcid.org/0000-0003-1238-2221

Juan C. Vázquez-Ucha

orcid.org/0000-0003-4949-0779

Laura Álvarez-Fraga

orcid.org/0000-0003-3920-5866

Lucía Ageitos

orcid.org/0000-0002-2422-3773

Soraya Rumbo-Feal

orcid.org/0000-0002-1796-1815

Marta Martínez-Guitián

orcid.org/0000-0002-3457-0613

Noelia Trigo-Tasende

orcid.org/0000-0001-5093-8994

Jaime Rodríguez

orcid.org/0000-0001-5348-6970

Germán Bou

orcid.org/0000-0001-8837-0062

Carlos Jiménez

orcid.org/0000-0003-2628-303X

Alejandro Beceiro

orcid.org/0000-0002-6340-7815

Margarita Poza

orcid.org/0000-0001-9423-7268

¥These authors have contributed equally to this work and share first authorship

\$These authors have contributed equally to this work and share last authorship

Specialty section:

This article was submitted to Infectious Agents and Disease,

a section of the journal

Frontiers in Microbiology

Received: 02 August 2021 Accepted: 16 September 2021 Published: 05 October 2021

\section{In-Depth Analysis of the Role of the} Acinetobactin Cluster in the Virulence of Acinetobacter baumannii

\author{
Kelly Conde-Pérez ${ }^{1,2 f \neq}$, Juan C. Vázquez-Ucha ${ }^{1 \neq \neq}$, Laura Álvarez-Fraga ${ }^{1,3 * t \neq}$, \\ Lucía Ageitos ${ }^{4 \dagger}$, Soraya Rumbo-Feal1,2†, Marta Martínez-Guitián ${ }^{1 \dagger}$, \\ Noelia Trigo-Tasende ${ }^{1 \dagger}$, Jaime Rodríguez ${ }^{4 \dagger}$, Germán Bou ${ }^{1 t}$, Carlos Jiménez ${ }^{4 t}$, \\ Alejandro Beceiro ${ }^{1+\S}$ and Margarita Poza ${ }^{1,2+\$}$
}

' Servicio de Microbiología del Complejo Hospitalario Universitario de A Coruña (CHUAC), Instituto de Investigación Biomédica de A Coruña (INIBIC), A Coruña, Spain, ${ }^{2}$ Microbiome and Health, Faculty of Science, University of A Coruña, A Coruña, Spain, ${ }^{3}$ School of Chemistry and Molecular Biosciences, University of Queensland, Brisbane, QLD, Australia, ${ }^{4}$ Centro de Investigaciones Científicas Avanzadas (CICA) y Departamento de Química, Facultad de Ciencias, Agrupación Estratégica CICA-INIBIC, Universidad de A Coruña, A Coruña, Spain

Acinetobacter baumannii is a multidrug-resistant pathogen that represents a serious threat to global health. $A$. baumannii possesses a wide range of virulence factors that contribute to the bacterial pathogenicity. Among them, the siderophore acinetobactin is one of the most important, being essential for the development of the infection. In this study we performed an in-depth analysis of the acinetobactin cluster in the strain A. baumannii ATCC 17978. For this purpose, nineteen individual isogenic mutant strains were generated, and further phenotypical analysis were performed. Individual mutants lacking the biosynthetic genes entA, basG, bas $C$, bas $D$, and basB showed a significant loss in virulence, due to the disruption in the acinetobactin production. Similarly, the gene bauA, coding for the acinetobactin receptor, was also found to be crucial for the bacterial pathogenesis. In addition, the analysis of the $\Delta b a s J / \Delta f b s B$ double mutant strain demonstrated the high level of genetic redundancy between siderophores where the role of specific genes of the acinetobactin cluster can be fulfilled by their fimsbactin redundant genes. Overall, this study highlights the essential role of ent $A$, basG , bas $C$, bas $D$, bas $B$ and bauA in the pathogenicity of $A$. baumannii and provides potential therapeutic targets for the design of new antivirulence agents against this microorganism.

Keywords: Acinetobacter baumannii, acinetobactin, fimsbactin, iron uptake, siderophore, virulence, mouse sepsis infection

\section{INTRODUCTION}

Acinetobacter baumannii is one of the most common nosocomial pathogens responsible for a wide range of concerning diseases, such as pneumonia, bacteremia or secondary meningitis (Wong et al., 2017). The rise of healthcare-associated infections caused by multidrug resistant strains of A. baumannii, together with the scarce development of new antimicrobials in the last decades, represents an important health threat (De Oliveira et al., 2020). In fact, A. baumannii, is one of 
the Gram-negative ESKAPE pathogens identified by the World Health Organization (WHO) as critical priority for antibiotic discovery (World Health Organization [WHO], 2017).

Although the main characteristic of this pathogen is its ability to acquire new antimicrobial resistance, it shows several mechanisms involved in virulence, persistence and stress adaptation that enhance its pathogenicity (Harding et al., 2018). Within this context, many researchers have focused their efforts in developing alternatives to conventional antibiotics, such as antivirulence agents, that can work alone or together with antibiotics to overcome A. baumannii infections (Dickey et al., 2017).

Iron is an essential micronutrient for bacteria to infect and multiply in tissues and body fluids of the host, playing an important role in pathogenesis. The mechanisms of bacterial iron acquisition include: (i) expression of transporters involved in the uptake of ferrous iron, such as the Feo system; (ii) extraction of heme-iron from hemoproteins; (iii) capture of iron from transferrin and lactoferrin; or (iv) synthesis of siderophores (Sheldon et al., 2016). Siderophores are high-affinity ironchelating molecules synthetized by microorganisms to scavenge extracellular ferric iron from the environment. Baumanoferrin, fimsbactin and preacinetobactin-acinetobactin (referred to as acinetobactin) are the most common siderophore systems detected in A. baumannii (Yamamoto et al., 1994; Proschak et al., 2013; Penwell et al., 2015). The most extensively studied is acinetobactin which is considered the major siderophore of A. baumannii and it is highly conserved among all A. baumannii strains (Antunes et al., 2011). All the genes required for the synthesis (bas $A-J)$, efflux $(\operatorname{bar} A / B)$ and uptake (bauA$F)$ of acinetobactin are located in a $26.5-\mathrm{kb}$ chromosomal region (Supplementary Figure 1A) (Mihara et al., 2004), with the exception of the entA homolog gene, found elsewhere in the chromosome (Penwell et al., 2012). Its biosynthesis follows the logic of a non-ribosomal peptide synthetase (NRPS) assembly system, where three precursors are bound in equimolar quantities into the preacinetobactin molecule: $N$-hydroxyhistamine, L-threonine and 2,3-dihydroxybenzoic acid (DHBA) (Figure 1A) (Yamamoto et al., 1994; Song and Kim, 2020). Once the preacinetobactin synthesis is completed, the siderophore is secreted to the extracellular space (Figure 1B) where two reactions can occur: (i) preacinetobactin stabilization by chelation of ferric iron or (ii) non-enzymatically and irreversibly isomerization to acinetobactin at $\mathrm{pH}>7$ (Shapiro and Wencewicz, 2016; Moynié et al., 2018). The fimsbactins A-F siderophores are present in a small fraction of the A. baumannii isolates (Antunes et al., 2011; Proschak et al., 2013). These siderophores, also derived from a NRPS assembly system, are structurally related to acinetobactin by the presence of catecholate, phenolate oxazoline, and hydroxamate metalbinding motifs (Proschak et al., 2013). Moreover, the cluster fbs $A-Q$, coding for the fimsbactins, consists of 18 genes with high functional similarity to those present in the acinetobactin cluster indicating redundancy between both siderophores pathways (Supplementary Figure 1B) (Dorsey et al., 2004; Mihara et al., 2004; Proschak et al., 2013). Although the expression of fimsbactins was shown to be enough to support the growth of
A. baumannii in serum, these siderophores are not required for survival during bacteremia (Sheldon and Skaar, 2020).

Indeed, acinetobactin has been shown to be essential for the virulence of $A$. baumannii during greater wax moth (Galleria mellonella) and murine bacteremia and pneumonia infections (Gaddy et al., 2012; Penwell et al., 2012; Martínez-Guitián et al., 2020; Sheldon and Skaar, 2020). However, no studies have explored the contribution of the entire collection of acinetobactin genes to the infectious process.

Herein, we have performed an in-depth analysis of the role of the acinetobactin cluster in the virulence of $A$. baumannii which allowed us to identify potential targets for the design of new antimicrobials against this pathogen.

\section{MATERIALS AND METHODS}

\section{Bacterial Strains and Culture Conditions}

All A. baumannii and Escherichia coli strains used in this study are listed in Table 1 . Bacteria were grown routinely at $37^{\circ} \mathrm{C}$ in solid and liquid Luria-Bertani (LB) medium and stored at $-80^{\circ} \mathrm{C}$ in LB broth containing $20 \%$ glycerol. When appropriate, media was supplemented with $50 \mu \mathrm{g} / \mathrm{mL}$ of kanamycin (Kan).

\section{Construction of Isogenic Mutant Derivative Strains}

All mutants were generated using the suicide vector pMo130 (Genbank: EU862243) as previously described (Álvarez-Fraga et al., 2016). Briefly, a PCR was performed to amplify both upstream and downstream regions flanking each gene of interest and cloned into the pMo130 vector. The plasmid constructions were electroporated into the wild-type strain A. baumannii ATCC 17978. Recombinant colonies representing the first crossover event were selected as previously described (Hamad et al., 2009). The second crossover event leading to gene knockout was confirmed by PCR followed by sequencing. All the primers used for the mutant construction are listed in the Supplementary Table 1.

\section{Growth Rate Analysis Under Normal and Iron-Limiting Conditions}

Growth rates were assessed by measuring the optical density (OD) of the A. baumannii ATCC 17978 parental strain and the mutant derivative strains in Mueller Hinton II (MH) medium, in the presence (iron-limiting conditions) or absence (normal conditions) of $0.2 \mathrm{mM}$ of the iron chelator 2,2' -bipyridyl (BIP), as previously described (Álvarez-Fraga et al., 2018). Growth was monitored at $\mathrm{OD}_{600}$ every $20 \mathrm{~min}$ until the late-log phase in 48-well plates using the Epoch 2 Microplate Spectrophotometer (BioTek Instruments, United States). The maximum specific growth rate $\left(\mu_{\max }\right)$ and the lag time $(\lambda)$ parameters were calculated using the single Gompertz growth curve model (Tjørve and Tjørve, 2017). The maximum specific growth rate parameter represents the slope of the tangent at the inflection point. The lag time parameter represents $x$ intercept of the $\mu_{\max }$ tangent and shows the time (h) to enter exponential 
A

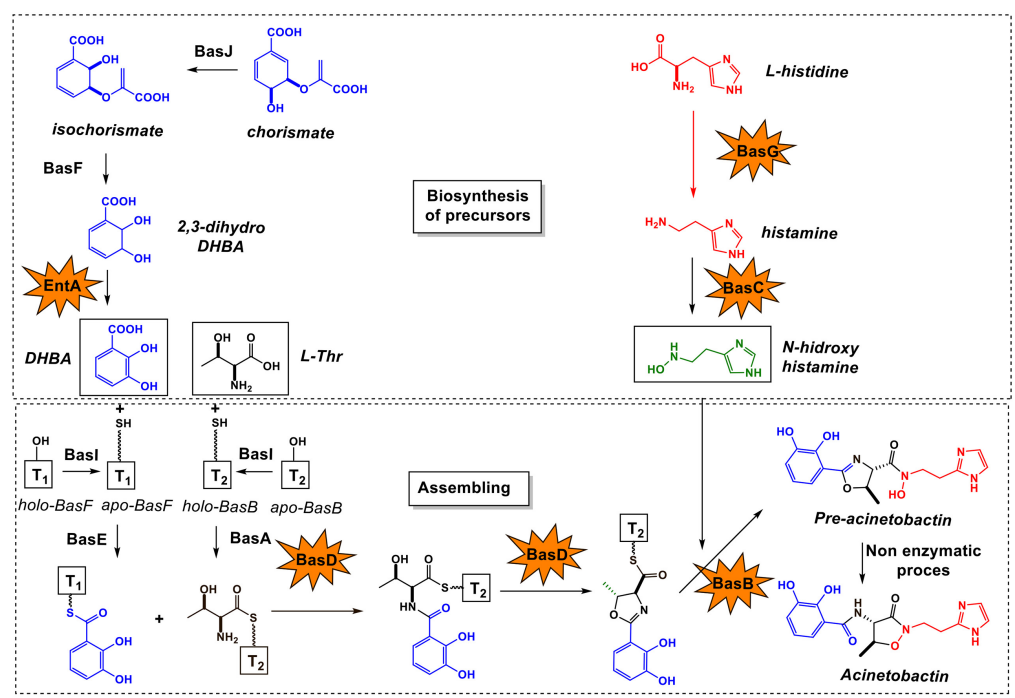

B

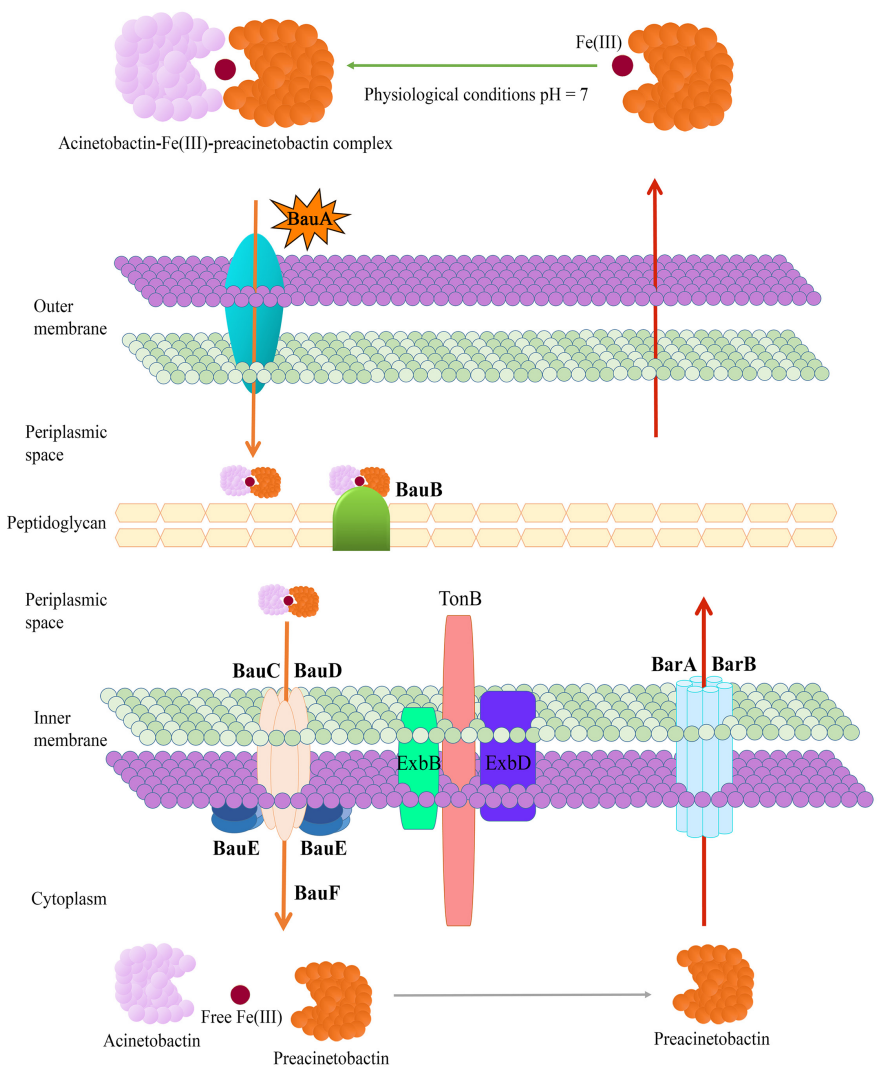

FIGURE 1 | Proposed (A) biosynthetic pathway and (B) transport mechanism of acinetobactin in A. baumannii. The six proteins found to be essential for the development of the bacteremia infection are marked with stars.

phase. Three independent biological replicates were carried out. Statistical analysis was performed using an unpaired, two tailed student's $t$-test.

\section{Murine Sepsis Model}

A murine sepsis model was used to evaluate the virulence of the A. baumannii ATCC 17978 parental strain and the isogenic mutant derivative strains as previously described (MartínezGuitián et al., 2019). Briefly, groups of 10 female BALB/c mice were inoculated intraperitoneally with approximately $7.5 \times 10^{7}$ colony forming units per mouse of exponentially grown cells and death was assessed during $168 \mathrm{~h}$ at 8 -h intervals. The survival curves were plotted using the Kaplan-Meier method and analysed using the log-rank (Mantel-Cox) test. All experiments 
TABLE 1 | Bacterial strains used in this work.

\begin{tabular}{|c|c|c|}
\hline Strain or plasmid & Relevant characteristics & Sources or references \\
\hline \multicolumn{3}{|l|}{ Strains } \\
\hline \multicolumn{3}{|l|}{ E. coli } \\
\hline TG1 & Used for DNA recombinant methods & Lucigen \\
\hline \multicolumn{3}{|l|}{ A. baumannii } \\
\hline ATCC 17978 & A. baumannii ATCC 17978 wild-type strain isolated from a fatal meningitis & American Type Culture Collection (ATCC) \\
\hline$\Delta$ bas」 & A1S_2372 gene deletion mutant from ATCC 17978 & This study \\
\hline$\Delta$ basl & A1S_2373 gene deletion mutant from ATCC 17978 & This study \\
\hline$\Delta$ basH & A1S_2374 gene deletion mutant from ATCC 17978 & This study \\
\hline$\Delta$ barB & A1S_2375 gene deletion mutant from ATCC 17978 & This study \\
\hline$\Delta b a r A$ & A1S_2376/77/78 gene deletion mutant from ATCC 17978 & This study \\
\hline$\Delta$ basG & A1S_2379 gene deletion mutant from ATCC 17978 & This study \\
\hline$\Delta$ basF & A1S_2380 gene deletion mutant from ATCC 17978 & This study \\
\hline$\Delta$ basE & A1S_2381 gene deletion mutant from ATCC 17978 & This study \\
\hline$\Delta$ bas $D$ & A1S_2382/83 gene deletion mutant from ATCC 17978 & This study \\
\hline$\Delta$ basC & A1S_2384 gene deletion mutant from ATCC 17978 & This study \\
\hline$\triangle b a u A$ & A1S_2385 gene deletion mutant from ATCC 17978 & This study \\
\hline$\Delta$ bauB & A1S_2386 gene deletion mutant from ATCC 17978 & This study \\
\hline$\Delta$ bauE & A1S_2387 gene deletion mutant from ATCC 17978 & This study \\
\hline$\Delta$ bauC & A1S_2388 gene deletion mutant from ATCC 17978 & This study \\
\hline$\Delta$ baud & A1S_2389 gene deletion mutant from ATCC 17978 & This study \\
\hline$\Delta$ basB & A1S_2390 gene deletion mutant from ATCC 17978 & Martínez-Guitián et al., 2020 \\
\hline$\triangle$ bas A & A1S_2391 gene deletion mutant from ATCC 17978 & This study \\
\hline$\Delta$ bauF & A1S_2392 gene deletion mutant from ATCC 17978 & This study \\
\hline$\Delta e n t A$ & A1S_2579 gene deletion mutant from ATCC 17978 & This study \\
\hline$\Delta b a s J / \Delta f b s B$ & A1S_2372 and A1S_2581 gene deletion double mutant from ATCC 17978 & This study \\
\hline$\Delta$ basF/ $\Delta f b s C$ & A1S_2380 and A1S_2580 gene deletion double mutant from ATCC 17978 & This study \\
\hline
\end{tabular}

were carried out with the approval of and in accordance with the regulatory guidelines and standards established by the Animal Ethics Committee (Hospital Universitario A Coruña, Spain, project code $\mathrm{P} 102)$.

\section{Chemical Analysis of the Siderophore Content of Acinetobacter baumannii Wild-Type and Mutant Strains}

The siderophore content of A. baumannii wild-type and the mutant strains was analyzed by using our SPE-HLB/HPLCHRMS methodology (Espada et al., 2011) adapted for the isolation of acinetobactin (Balado et al., 2015) and detecting the presence of iron(III) chelating compounds using the Chrome Azurol-S Liquid (CAS) assay. Briefly, bacteria were grown at $37^{\circ} \mathrm{C}$ in $\mathrm{M} 9$ minimal media supplemented with $0.2 \%$ casamino acids and $0.4 \%$ glucose until an $\mathrm{OD}_{600}=1.0$. Subsequently, the bacterial suspensions were pelleted, filtered and the resultant cellfree supernatants were freeze-dried to obtain $2.5 \mathrm{~g}$ of a residue. One gram of this material was dissolved in milli-Q water $(1 \mathrm{~mL})$, loaded in an OASIS HLB cartridge (6 g, $35 \mathrm{~cm} 3$, Waters), which was previously conditioned and equilibrated with $120 \mathrm{~mL}$ of acetonitrile (solvent B) and water (solvent A), each containing $0.1 \%$ TFA (v/v), and fractionated with 1:0, 9:1, 8:2, 7:3, and 0:1 of $\mathrm{A}: \mathrm{B}(\mathrm{v} / \mathrm{v})$ to give ABLH1-5 fractions, respectively. CAS-positive fractions were further analyzed by HPLC (Thermo Scientific) coupled to a PDA detector, monitoring the absorbance at $\lambda=254$, 280 and $313 \mathrm{~nm}$, and to a MSQ plus mass spectrometer in full positive ion mode. The analysis was carried out using a Discovery HS-F5 column $(100 \times 4.6 \mathrm{~m}, 5 \mu \mathrm{m})$, with a flow of $1 \mathrm{~mL} / \mathrm{min}$ and the following gradient conditions: acetonitrile (solvent B) and water (solvent A), each containing $0.1 \%$ TFA (v/v), $40 \mathrm{~min}$ from 10 to $50 \%$ of $\mathrm{B}, 5 \mathrm{~min}$ from 50 to $100 \%$ of $\mathrm{B}$, a $5 \mathrm{~min}$ of an isocratic step at $100 \%$ of $\mathrm{B}, 5 \mathrm{~min}$ from 100 to $10 \%$ of $\mathrm{B}$ and final $5 \mathrm{~min}$ of an isocratic step at $10 \%$ of B. HPLC/HRMS analysis of the ABLH3 fraction showed the presence of a chromatographic peak with a $\mathrm{rt}=11.75 \mathrm{~min}$, which presented a $[\mathrm{M}+\mathrm{H}]^{+}$adduct in its corresponding $(+)$HR-ESIMS at $m / z 347.1344$ that agreed to that of acinetobactin (calcd. for $\mathrm{C}_{16} \mathrm{H}_{19} \mathrm{~N}_{4} \mathrm{O}_{5}, \mathrm{~m} / z$ 347.1350). In parallel, fimsbactins $\mathrm{A}$ and $\mathrm{F}$ were detected in the chromatographic peak with a $\mathrm{rt}=19.4 \mathrm{~min}$ of ABLH5 by displaying the $[\mathrm{M}+\mathrm{H}]^{+}$ adducts at $m / z 575.1956$ (calcd. for $\mathrm{C}_{26} \mathrm{H}_{31} \mathrm{~N}_{4} \mathrm{O}_{11}, 575.1989$ ) and $m / z 439.1803$ (calcd. for $\mathrm{C}_{19} \mathrm{H}_{27} \mathrm{~N}_{4} \mathrm{O}_{8}, 439.1829$ ) in the (+)-HR-ESIMS, respectively. Analogs of fimsbactins A and F, where the oxazoline ring is opened, were also identified in the chromatographic peak with a $\mathrm{rt}=13.4 \mathrm{~min}$ by showing the $[\mathrm{M}+\mathrm{H}]^{+}$adducts at $m / z 593.2063$ (calcd. for $\mathrm{C}_{26} \mathrm{H}_{33} \mathrm{~N}_{4} \mathrm{O}_{12}$, 575.2089) and $m / z 457.1909$ (calcd. for $\mathrm{C}_{19} \mathrm{H}_{29} \mathrm{~N}_{4} \mathrm{O}_{8}, 457.1229$ ) in their (+)-HR-ESIMS. These analogs were formed from fimsbactins $\mathrm{A}$ and $\mathrm{F}$ due to the acidic conditions used for separation. Indeed, these compounds were not obtained when 
the same SPE-HLB/HPLC-MS methodology was used avoiding acidic conditions.

\section{RESULTS}

\section{Specific Genes of the Acinetobactin Cluster Are Relevant for the Growth of Acinetobacter baumannii ATCC 17978 Under Iron-Limiting Conditions}

To determine the contribution of each gene of the acinetobactin cluster to the growth of A. baumannii ATCC 17978, a total of 19 individual isogenic mutant strains were generated (Figure 1, Table 1, Supplementary Figure 1, and Supplementary Table 2) and growth curves were performed in absence (normal conditions) and presence (iron-limiting conditions) of the iron (III) 2,2'- bipyridyl (BIP). The eleven mutant strains lacking the genes involved in the biosynthesis of acinetobactin were classified in three different groups: (i) genes involved in the synthesis of the DHBA precursor (basJ, basF, and entA), (ii) genes involved in the synthesis of the $N$-hydroxyhistamine precursor (bas $G$ and bas $C$ ) and (iii) genes involved in the modification and assembly of the acinetobactin precursors into the final molecule (basI, basH, basD, basB, basA, and basE) (Figure 1A). The growth curves under iron-limiting conditions of the strains lacking the genes involved in the DHBA synthesis revealed that the $\triangle e n t A$ mutant was impaired for growth, with a lower maximum specific growth rate $\left(\mu_{\max }\right)(0.24, P=0.0008)$ and a higher lag time $(\lambda)(4.2, P=0.0129)$ than the parental strain ( $\mu_{\max }=0.74$ and $\lambda=1.36$ ). The deletion of basF also resulted in a slightly decrease of $\mu_{\max }(0.49, P=0.0119)$ and $\lambda(0.81$, $P=0.0423$ ) (Figure $2 \mathrm{~A}$ and Table 2). The mutant strains $\Delta$ bas $G$ and $\Delta$ bas $C$, lacking genes involved in the synthesis of the $N$-hydroxyhistamine precursor, displayed a significant reduction in the $\mu_{\max }(0.48, P=0.0084$ and $0.43, P=0.0061$; respectively) and an increase in the $\lambda(3.7, P=0.0002$ and 3.9, $P=0.0001$; respectively) when compared to the wild-type strain (Figure $2 \mathbf{B}$ and Table 2). Among the mutants belonging to the third group, $\triangle b a s D, \Delta b a s B$, and $\triangle$ bas $A$ grew poorly under iron-limitation conditions, showing significant lower $\mu_{\max }(0.49, P=0.01 ; 0.46$, $P=0.0072$ and $0.30, P=0.0012$; respectively). The $\triangle b a s D$ and $\triangle$ bas $B$ mutants also shown higher lag times $(\lambda=3.99, \mathrm{P}=0.0002$ and $\lambda=4.86, P<0.0001$; respectively) compared to the wildtype strain (Figure 2C and Table 2). No significant differences in growth kinetics were observed in the other isogenic mutant strains $\left[P(\lambda)=0.16-0.82, P\left(\mu_{\max }\right)=0.09-0.74\right]$ compared to the wild-type strain ATCC 17978 (Figure 2). Under normal conditions, the eleven isogenic mutant strains showed similar growth abilities compared to the wild-type strain ATCC 17978 (Supplementary Figure 2A).

In parallel, we performed growth curves with the eight isogenic mutant derivative strains, lacking each of the influx and efflux related genes, under both normal and ironlimiting conditions. As it was observed in the mutant strains related to the acinetobactin biosynthesis, no significant differences were detected between the isogenic mutant strains and the wild-type strain under normal growth conditions (Supplementary Figure 2B). Nevertheless, under iron-limited conditions, the deletion of the genes barA, barB (efflux), and bauC (uptake) resulted in a significant growth inhibition shown as a reduction in the $\mu_{\max }(0.34, P=0.0018 ; 0.30, P=0.0013$ and $0.55, P=0.0241$; respectively) and an increase in the $\lambda(3.99$, $P=0.0001 ; 4.11, P<0.0001$ and $2.3, P<0.0051$; respectively) (Figure 3 and Table 2). We also observed a partial reduction of the growth of $\triangle$ bauD $\left(\mu_{\max }=0.56, P=0.0320\right), \Delta b a u E$ $\left(\mu_{\max }=0.49, P=0.0128\right)$ and $\Delta$ bauB $(\lambda=1.91, P<0.0321)$ mutant strains growth. No significant differences in growth kinetics were observed in the other isogenic mutant strains $[P$ $\left.(\lambda)=0.22-0.51, P\left(\mu_{\max }\right)=0.06-0.86\right]$ (Figure 3).

\section{Six Genes of the Acinetobactin Cluster Are Essential for the Virulence in vivo}

It has been previously showed that acinetobactin plays an important role in the virulence of A. baumannii (Gaddy et al., 2012; Penwell et al., 2012; Martínez-Guitián et al., 2020; Sheldon and Skaar, 2020). However, it remains unclear which genes of the acinetobactin cluster are essential for the development of the infection. To elucidate this, a murine sepsis model was performed with the wild-type and the 19 mutant derivative strains. Among the mutant strains lacking genes involved in the biosynthesis of acinetobactin, the mice infected with $\Delta$ entA (90\% survival, $P=0.0009), \Delta$ basG (90\% survival, $P=0.0004), \Delta$ basC $(90 \%$ survival, $P=0.0009), \Delta$ basD (90\% survival, $P=0.0004)$, and $\triangle$ basB $(100 \%$ survival, $P<0.0001)$ mutant strains showed survival rates significantly higher compared with those of the mice infected with the parental strain (10\% survival) (Figures 4A-C and Table 2). No significant differences in mice survival were observed in the other isogenic mutant strains $(P=0.3068-0.9716)$ (Figures 4A-C).

Among the mutant strains lacking genes involved in the transport of acinetobactin, only mice infected with the $\triangle b a u A$ strain showed a significant increase in the survival rate $(80 \%$ survival, $P=0.0039$ ) compared with those mice infected with the parental strain (10\% survival) (Figure 4D and Table 2). No significant differences in mice survival were observed in the other isogenic mutant strains $(P=0.1926-0.9484)$ (Figure 4D).

\section{Analysis of the Siderophore-Content of Acinetobacter baumannii Wild-Type and Mutant Strains}

To confirm whether the deletion of specific genes in A. baumannii ATCC 17978 caused a disruption in the biosynthesis or transport of acinetobactin, we analysed the presence of this siderophore in eight isogenic mutant strains (Table 2): $\triangle b a s B, \Delta$ basG, $\Delta$ bas $C, \Delta b a s D$, and $\Delta$ basJ, related to the biosynthesis; $\triangle$ bauA and $\triangle b a u B$, related to the influx and $\triangle b a r B$, related to the efflux of acinetobactin. We employed a bio-guided fractionation based on the SPE-HLB/HPLC-MS methodology described by Balado et al. (2015), using the colorimetric CAS liquid assay for the detection of iron(III)chelating compounds (Supplementary Figure 3A). Thus, the cell-free supernatants of interest were freeze-dried and 

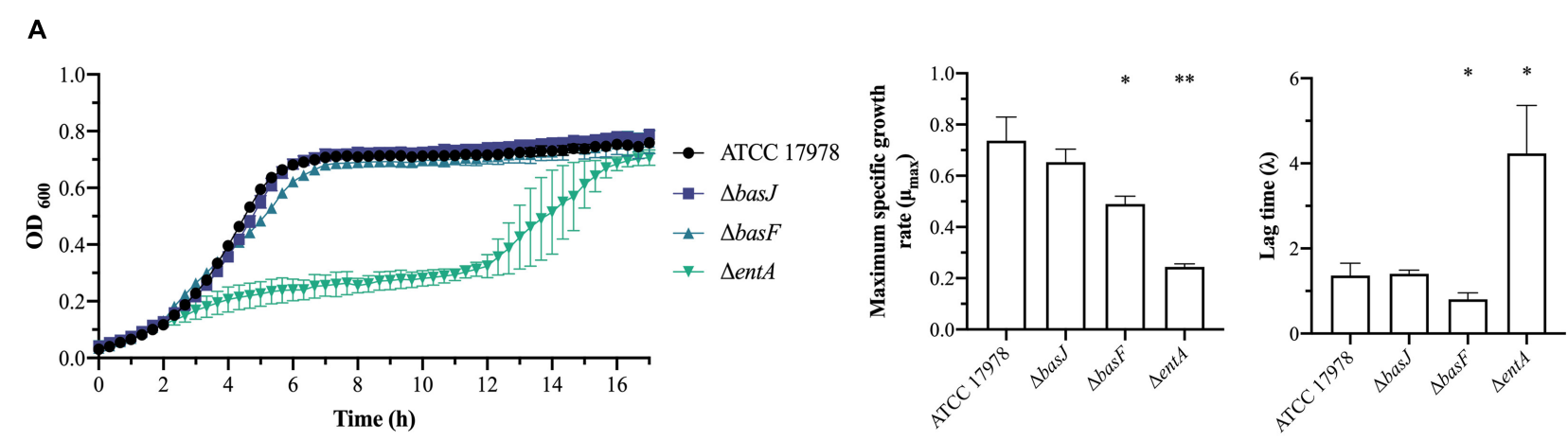

B
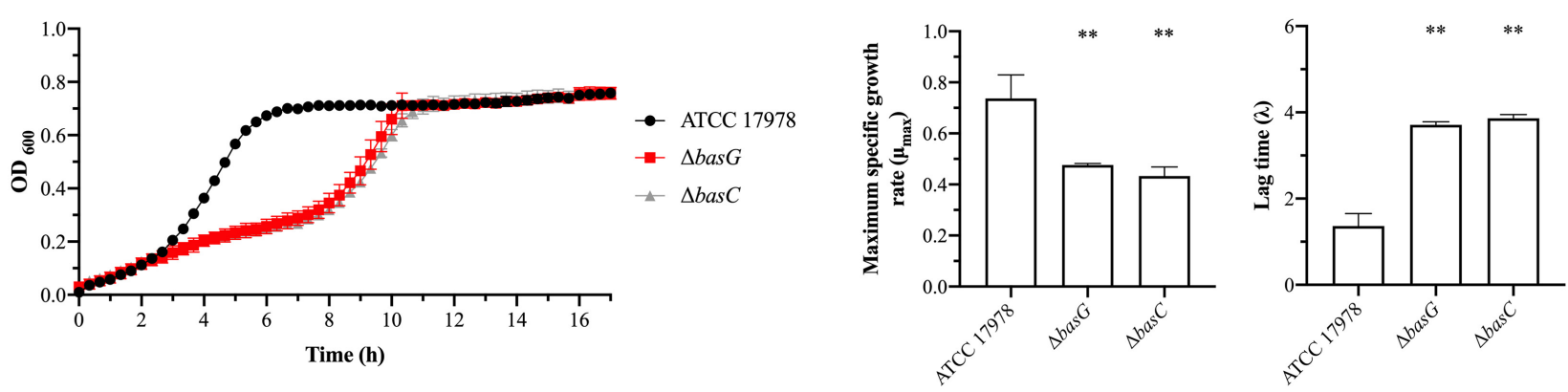

C
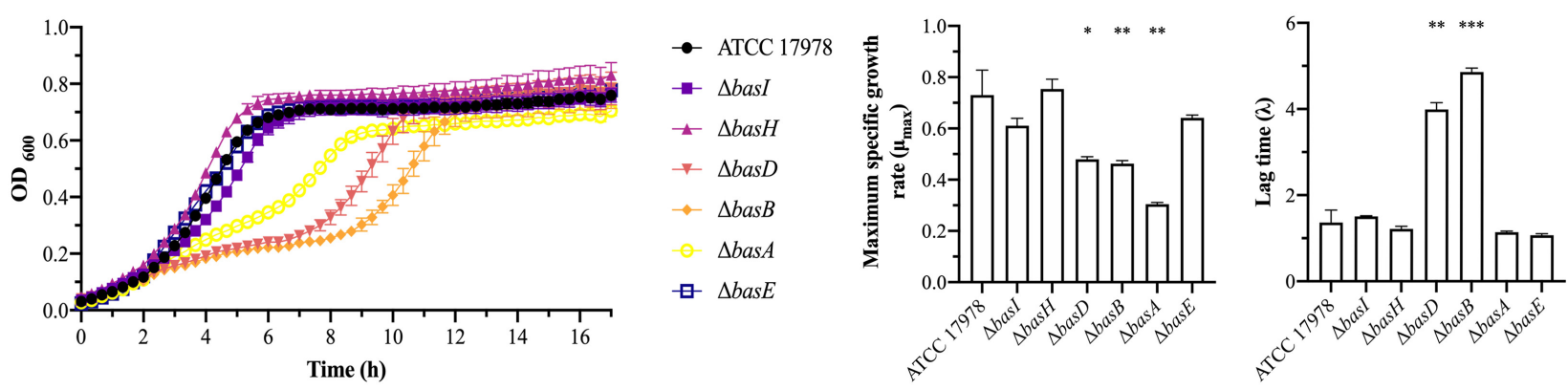

FIGURE 2 | Growth curves and growth kinetics of A. baumannii ATCC 17978 and its isogenic mutant derivative lacking (A) genes involved in the synthesis of the DHBA precursor, (B) genes involved in the synthesis of the $N$-hydroxyhistamine precursor and (C) genes involved in the modification and assembly of the acinetobactin precursors into the final molecule. The growth curves were performed under iron-limiting conditions. Three independent biological replicates were performed. Unpaired student $t$ test was used for the statistical analysis of the growth kinetics $\left({ }^{*} P<0.05\right.$; ${ }^{* *} P<0.01$; $\left.{ }^{* * *} P<0.0001\right)$.

fractionated by solid-phase extraction (SPE) using hydrophiliclipophilic balance (HLB) cartridges (Figure 5A). HPLC/HRMS analysis of the CAS-positive fractions obtained from the wildtype strain allowed us to detect acinetobactin and fimsbactins A and $\mathrm{F}$ (Figure 5A). Specifically, acinetobactin was localized in the chromatographic peak with $\mathrm{rt}=11.75 \mathrm{~min}$ of the fraction ABLH3 eluted from the HLB cartridge with 8:2 of $\mathrm{H}_{2} \mathrm{O}: \mathrm{CH}_{3} \mathrm{CN}$ $(\mathrm{v} / \mathrm{v})$, each containing $0.1 \%$ TFA $(\mathrm{v} / \mathrm{v})$, showing a $[\mathrm{M}+\mathrm{H}]^{+}$ ion at $\mathrm{m} / \mathrm{z} 347$ in its corresponding MS (Figures 5B,C and Supplementary Figure 4). On the other hand, fimsbactin A and F were detected in the chromatographic peak with $\mathrm{rt}=19.4 \mathrm{~min}$ of ABHL5 eluted with $100 \%$ of $\mathrm{CH}_{3} \mathrm{CN}$, containing $0.1 \%$ TFA, displaying a $[\mathrm{M}+\mathrm{H}]^{+}$ion at $\mathrm{m} / z 575$ and 439 , respectively, in their MS (Supplementary Figures 5, 6). Due to the acidic conditions of the methodology, two analogs of fimsbactin A and F, having an opened oxazoline ring, were also found in the chromatographic peak with $\mathrm{rt}=13.4 \mathrm{~min}$ of this fraction, displaying a $[\mathrm{M}+\mathrm{H}]^{+}$ion at $\mathrm{m} / z 593$ and 457 , respectively (Supplementary Figures 5, 7). This was confirmed after the analysis of the analogous fraction (ABLHWA5) obtained under non-acidic conditions (Supplementary Figures 3B, 8).

Finally, comparison of the HPLC chromatographic profiles of ABHL3 and ABHL5 fractions from the parental strain and the former selected mutant derivative strains, revealed that those mutants lacking genes involved in the biosynthesis of acinetobactin, except for $\Delta$ basJ, were not able to produce acinetobactin (Figure 5B, Table 2, and Supplementary Figure 9). However, the five mutant strains displayed the presence of fimsbactins $\mathrm{A}$ and $\mathrm{F}$ in their ABHL5 fractions (Table 2 and Supplementary Figure 10). In parallel, the mutant strains lacking genes involved in the influx and efflux of acinetobactin did not show any difference 
TABLE 2 | Assays performed with the 21 isogenic mutant derivative strains.

\begin{tabular}{|c|c|c|c|c|c|c|c|c|}
\hline \multirow[t]{2}{*}{ Mutant strains } & \multirow[t]{2}{*}{ Gene } & \multirow[t]{2}{*}{ Function } & \multirow{2}{*}{$\begin{array}{c}\text { Fitness under } \\
\text { iron-limiting } \\
\text { conditions ( } \mu \text { max) }\end{array}$} & \multirow{2}{*}{$\begin{array}{l}\text { Fitness under } \\
\text { iron-limiting } \\
\text { conditions }(\lambda)\end{array}$} & \multirow{2}{*}{$\begin{array}{l}\text { Mice survival during } \\
\text { sepsis }\end{array}$} & \multicolumn{3}{|c|}{ SPE-HLB/HPLC-MS } \\
\hline & & & & & & Acinetobactin & Fimsbactin A & Fimsbactin F \\
\hline
\end{tabular}

Mutant strains lacking genes involved in the acinetobactin biosynthesis

$\begin{array}{lll}\Delta \text { basJ } & \text { A1S_2372 } & \text { DHBA synthesis } \\ \Delta \text { basF } & \text { A1S_2380 } & \\ \Delta \text { entA } & \text { A1S_2579 } & \\ \Delta \text { basG } & \text { A1S_2379 } & \text { N-hydroxyhistamine synthesis } \\ \Delta \text { basC } & \text { A1S_2384 } & \\ \Delta \text { basl } & \text { A1S_2373 } & \text { NRPS assembly system } \\ \Delta \text { basH } & \text { A1S_2374 } & \\ \Delta \text { basD } & \text { A1S_2382/83 } & \\ \Delta \text { basB } & \text { A1S_2390 } & \\ \Delta \text { basA } & \text { A1S_2391 } & \\ \Delta \text { basE } & \text { A1S_2381 } & \end{array}$

$\begin{array}{cc} & \\ - & - \\ + & +^{\mathrm{a}} \\ ++ & + \\ ++ & ++ \\ ++ & ++ \\ - & - \\ - & - \\ + & ++ \\ ++ & +++ \\ ++ & - \\ - & - \\ & \\ ++ & ++ \\ ++ & +++ \\ - & - \\ - & + \\ + & ++ \\ + & - \\ + & - \\ - & -\end{array}$

Mutant strains lacking genes involved in acinetobactin transport

$\begin{array}{llllc}\Delta \text { barA } & \text { A1S_2376/77/78 } & \text { Efflux } & ++ & ++ \\ \Delta \text { barB } & \text { A1S_2375 } & & ++ & ++ \\ \Delta \text { bauA } & \text { A1S_2385 } & \text { Influx } & - & - \\ \Delta \text { bauB } & \text { A1S_2386 } & & - & + \\ \Delta \text { bauC } & \text { A1S_2388 } & & + & ++ \\ \Delta \text { baul } & \text { A1S_2389 } & & + & - \\ \Delta \text { bauE } & \text { A1S_2387 } & & + & - \\ \Delta \text { bauF } & \text { A1S_2392 } & & - & -\end{array}$

\section{Double mutant strains}

\begin{tabular}{|c|c|c|c|c|c|c|c|c|}
\hline$\Delta b a s J / \Delta f b s B$ & A1S_2372/A1S_2581 & DHBA synthesis & ++ & ++ & +++ & n.d. & n.d & n.d \\
\hline$\Delta b a s F / \Delta f b s C$ & A1S_2380/A1S_2580 & & ++ & +++ & +++ & n.a & n.a & n.a \\
\hline
\end{tabular}



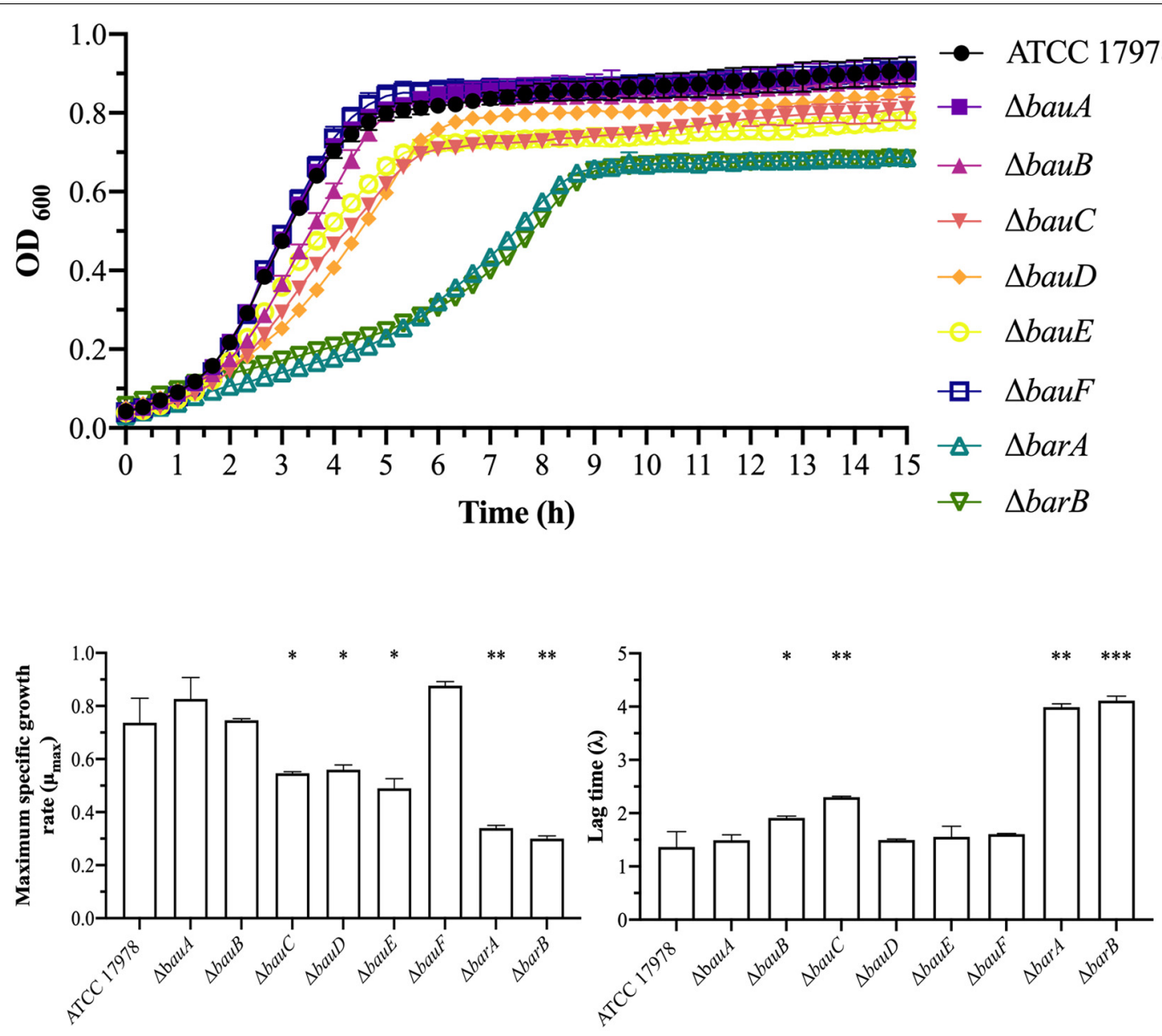

FIGURE 3 | Growth curves and growth kinetics of $A$. baumannii ATCC 17978 and the isogenic mutant derivative strains lacking genes involved in the transport of acinetobactin. The growth curves were performed under iron-limiting conditions. Three independent biological replicates were performed. Unpaired student $t$ test was used for the statistical analysis of the growth kinetics $\left({ }^{*} P<0.05\right.$; ${ }^{* *} P<0.01$; ${ }^{* * *} P<0.0001$ ).

in comparison to the parental strain in none of the fractions (Supplementary Figures 11-14).

\section{Specific Genes of the Fimsbactin Cluster Could Contribute to the Acinetobactin Biosynthesis}

Our results showed that the genes bas $G$ and basC, involved in the biosynthesis of the $N$-hydroxyhistamine precursor, and the genes bas $D$ and $b a s B$, involved in the assembly of the precursors, are essential for the biosynthesis of acinetobactin and therefore, they are crucial for the virulence of A. baumannii in vivo. However, the deletion of basJ gene which is involved in the biosynthesis of the DHBA precursor did not have any effect in the biosynthesis of acinetobactin and in the virulence of the bacterium. Taking into account that DHBA is also a fimsbactin precursor, our data suggest that in the absence of specific genes of the acinetobactin cluster, A. baumannii ATCC 17978 is able to successfully synthetize acinetobactin using redundant genes from the fimsbactin cluster.
To explore this hypothesis, we performed an in-depth analysis of both clusters using BLAST (see Supplementary Figure 1 for cluster organization and Supplementary Table 2 for gene description). This analysis showed that all the genes (except for entA) belonging to the acinetobactin cluster involved in the DHBA biosynthesis and the NRPS assembly have a potential redundant gene in the fimsbactin cluster (Supplementary Table 3). To further investigate this genetic redundancy, basJ and basF genes were selected and two double mutant strains were generated lacking both redundant genes of the acinetobactin and the fimsbactin clusters ( $\triangle$ basJ/ $\Delta f b s B$ and $\Delta b a s F / \Delta f b s C$ ) (Table 1).

Growth curves under iron-limiting conditions showed a significant decrease in the growth abilities of both $\Delta$ bas J/ $\Delta \mathrm{fbs} B$ $\left(\mu_{\max }=0.24, P=0.001\right.$ and $\left.\lambda=8.94, P=0.0009\right)$ and $\Delta$ basF/ $\triangle f b s C\left(\mu_{\max }=0.38, P=0.006\right.$ and $\left.\lambda=9.65, P<0.0001\right)$ mutant strains compared to the parental strain ATCC 17978 $\left(\mu_{\max }=0.74\right.$ and $\lambda=1.36$ ) (Figure 6A and Table 2). However, no significant differences were detected between the isogenic double mutant strains and the wild-type strain under normal growth conditions (Supplementary Figure 2C). 


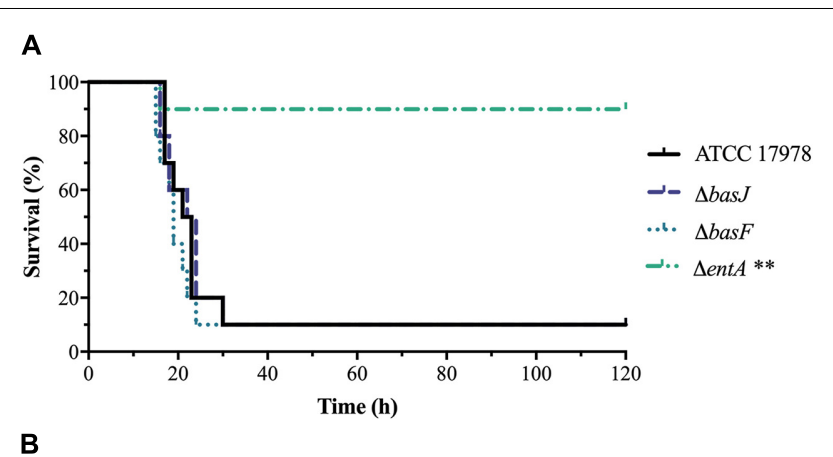

B

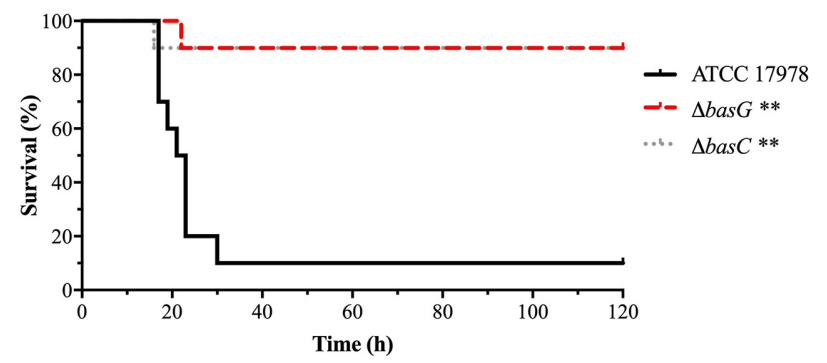

C

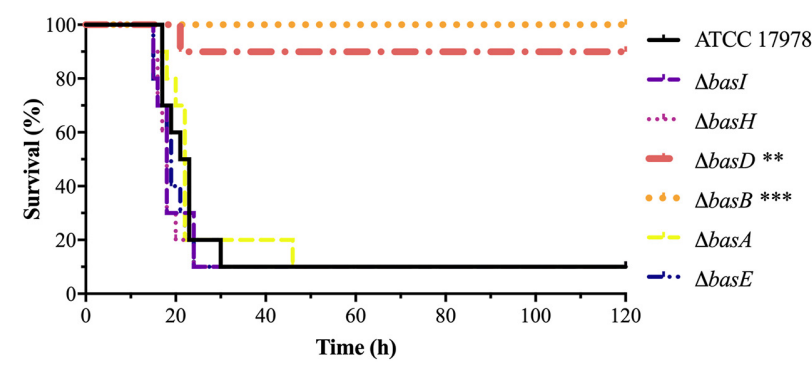

D

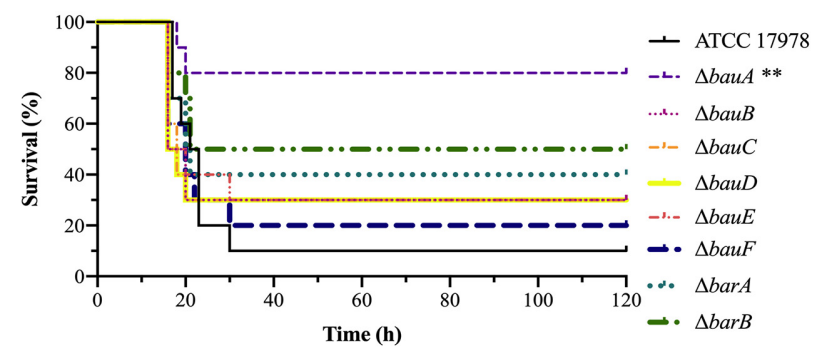

FIGURE 4 | Sepsis infection in mice. Survival of BALB/c mice $(n=10$ per group) after bacteremia infection with $A$. baumannii ATCC 17978 and the isogenic mutant derivative strains lacking (A) genes involved in the synthesis of the DHBA precursor, (B) genes involved in the synthesis of the $\mathrm{N}$-hydroxyhistamine precursor, (C) genes involved in the modification and assembly of the acinetobactin precursors into the final molecule and (D) genes involved in the acinetobactin transport The log-rank (Mantel-Cox) test was used for statistical analysis $\left({ }^{* *} P<0.01,{ }^{* * *} P<0.0001\right)$.

Furthermore, a murine sepsis model was performed with the ATCC 17978 parental strain and the two double isogenic mutant strains. Mice infected with $\Delta b a s J / \Delta f b s B$ and $\Delta b a s F / \Delta f b s C$ strains displayed a significantly increase of the survival rate $(100 \%$ survival, $P<0.0001$ ) in relation to those infected with the ATCC 17978 parental strain (10\% survival) (Figure 6B and Table 2).
Finally, the siderophore-content of the double mutant $\Delta b a s J / \Delta f b s B$ was studied using our SPE-HLB/HPLC/MS methodology, revealing complete inhibition of acinetobactin and fimsbactins production by lack of detection of these siderophores in the HPLC/MS analysis (Table 2 and Supplementary Figures 13, 14).

\section{DISCUSSION}

In the last decades, the emergence of A. baumannii multidrugresistant strains has become a worldwide concerning problem derived from the scarcity of effective therapeutic options against this bacterium. Hence, the World Health Organization (WHO) included $A$. baumannii as a critical priority pathogen claiming an urgent need of efficient alternatives to the known antibiotics (World Health Organization [WHO], 2017). Within this context, the search and identification of new therapeutic targets in A. baumannii have become a priority.

The pathogenesis success of A. baumannii is partially linked to the synthesis of active siderophores that supply the iron needed for its essential role in crucial metabolic events. Among all the siderophore systems identified, acinetobactin is considered the major siderophore of this bacterium (Yamamoto et al., 1994). Since then, several studies have focused on unraveling its regulation, chelating mechanisms and its role in the virulence of the bacterium (Mihara et al., 2004; Gaddy et al., 2012; Shapiro and Wencewicz, 2016; Sheldon and Skaar, 2020). After confirming that acinetobactin-related metabolism is a crucial virulence factor and that it is highly conserved among A. baumannii strains, the iron(III) uptake system mediated by acinetobactin has been proposed as a potential therapeutic target to combat this multidrug-resistant pathogen (Antunes et al., 2011; Gaddy et al., 2012; Sheldon and Skaar, 2020). However, the contribution of each individual gene involved in the acinetobactin metabolism in the infectious process is still unclear. Thus, we have performed an in-depth analysis of the acinetobactin gene cluster by conducting different phenotypical assays with the well-known strain A. baumannii ATCC 17978 and 19 isogenic mutant derivative strains lacking genes involved in the biosynthesis and transport of acinetobactin.

Three different siderophore-mediated iron uptake systems (acinetobactin, baumanoferrin and fimsbactin) were identified from the reference strain A. baumannii ATCC 17978, which corresponding gene clusters were found upregulated under in vitro iron-limiting conditions and in vivo infection (Eijkelkamp et al., 2011; Murray et al., 2017; Martínez-Guitián et al., 2020). Acinetobactin and fimsbactins are synthesized through non-ribosomal peptide synthetase (NRPS) assembly systems, sharing the DHBA and L-threonine precursors (Proschak et al., 2013; Song and Kim, 2020). This could explain the high level of genetic redundancy between both clusters where most of the genes involved in the biosynthesis of the acinetobactin have a potential redundant gene in the fimsbactin cluster. Hence, although fimsbactins are only present in a small percentage of $A$. baumannii strains, fimsbactin genes could complement the inactivation of some acinetobactin genes, 


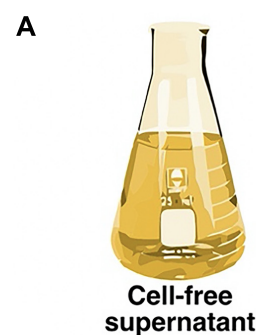

B

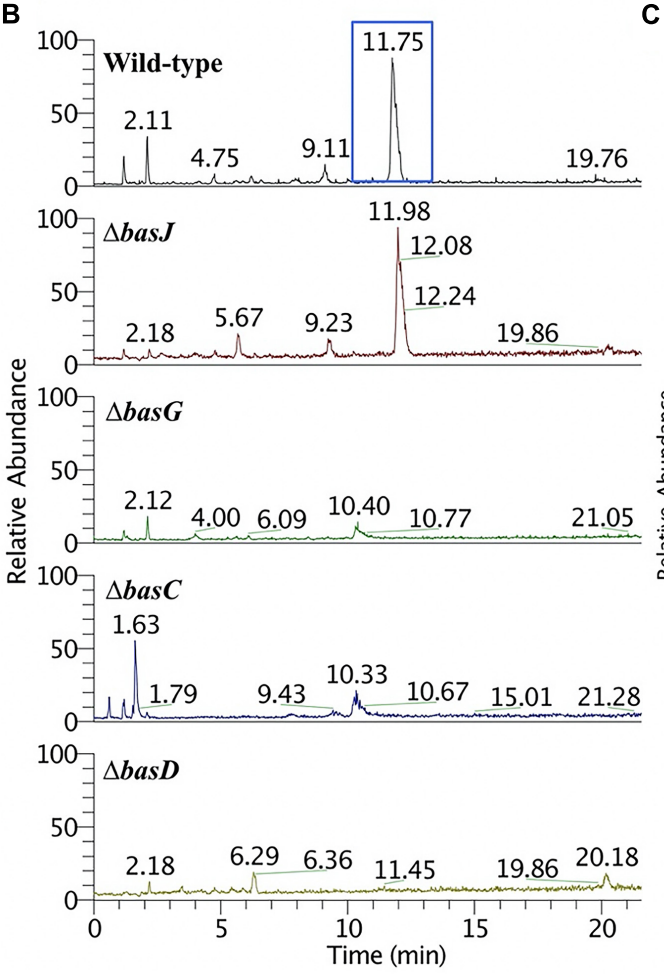

C

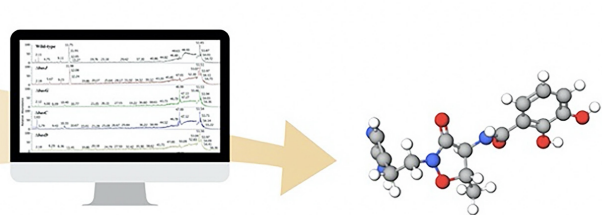

HPLC/MS analysis

Siderophore determination

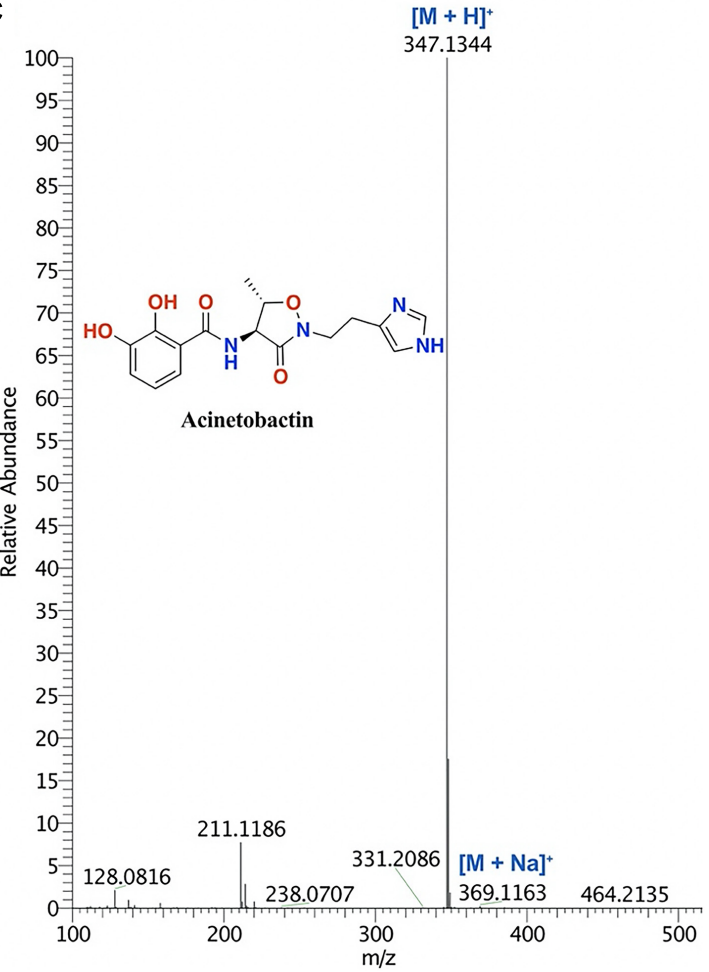

FIGURE 5 | (A) Schematic representation of the siderophore-content isolation SPE-HLB/HPLC-MS methodology carried out in the cell-free supernatant of A. baumannii wild-type and mutant strains. (B) Comparison of the total ion current (TIC) chromatographic profiles of the ABLH3 fraction of $A$. baumannii wild type, in which acinetobactin was detected at $\mathrm{rt}=11.75 \mathrm{~min}$, to that of five mutant strains with depleted genes involved in the biosynthesis of acinetobactin. (C) High resolution mass spectrum of acinetobactin detected in the chromatographic peak at rt $=11.75$ min of the ABLH3 fraction obtained from the $A$. baumannii wild-type strain.

suggesting a redundancy in both pathways. Within this context, A. baumannii ATCC 17978 confers the perfect background for the present study.

Bioinformatic analysis of the acinetobactin gene cluster showed that basG, bas $C$ and entA genes do not have a potential redundant gene. The lack of redundant bas $G$ and bas $C$ genes in the fimsbactin cluster is easily explained since these genes are involved in the biosynthesis of the precursor of acinetobactin $N$-hydroxyhistamine (Shapiro and Wencewicz, 2016) and this moiety is not present in the fimsbactins. On the other hand, the ent $A$ gene is always located outside of the acinetobactin cluster, varying its location between strains. In A. baumannii ATCC 17978, acinetobactin and fimsbactins share the gene entA, which is located in the fimsbactin cluster (Penwell et al., 2012). The individual deletion of these three genes resulted in a drastic reduction of the virulence when compared to the wild-type strain. Our results agreed with a recent study published by Sheldon and Skaar where they demonstrated that the deletion of gene bas $G$ impairs growth on human serum, transferrin or lactoferrin as sole iron sources, and severely attenuates survival of A. baumannii ATCC 17978 in a murine bacteremia model (Sheldon and Skaar, 2020).

Although Dorsey et al. predicted that basC gene had an essential function in the biosynthesis of the acinetobactin on the basis of its involvement in the synthesis of $N$-hydroxyhistamine (Dorsey et al., 2004), this hypothesis was never investigated until now. Siderophore-content analysis of the cell free supernatants of both $\triangle b a s G$ and $\triangle b a s C$ using our SPE-HLB/HPLC-MS methodology showed that the deletion of these genes resulted in the complete inhibition of acinetobactin production. The low virulent phenotype of these two mutants could be related to the lack of the siderophore. As the previous case, it would be expected 


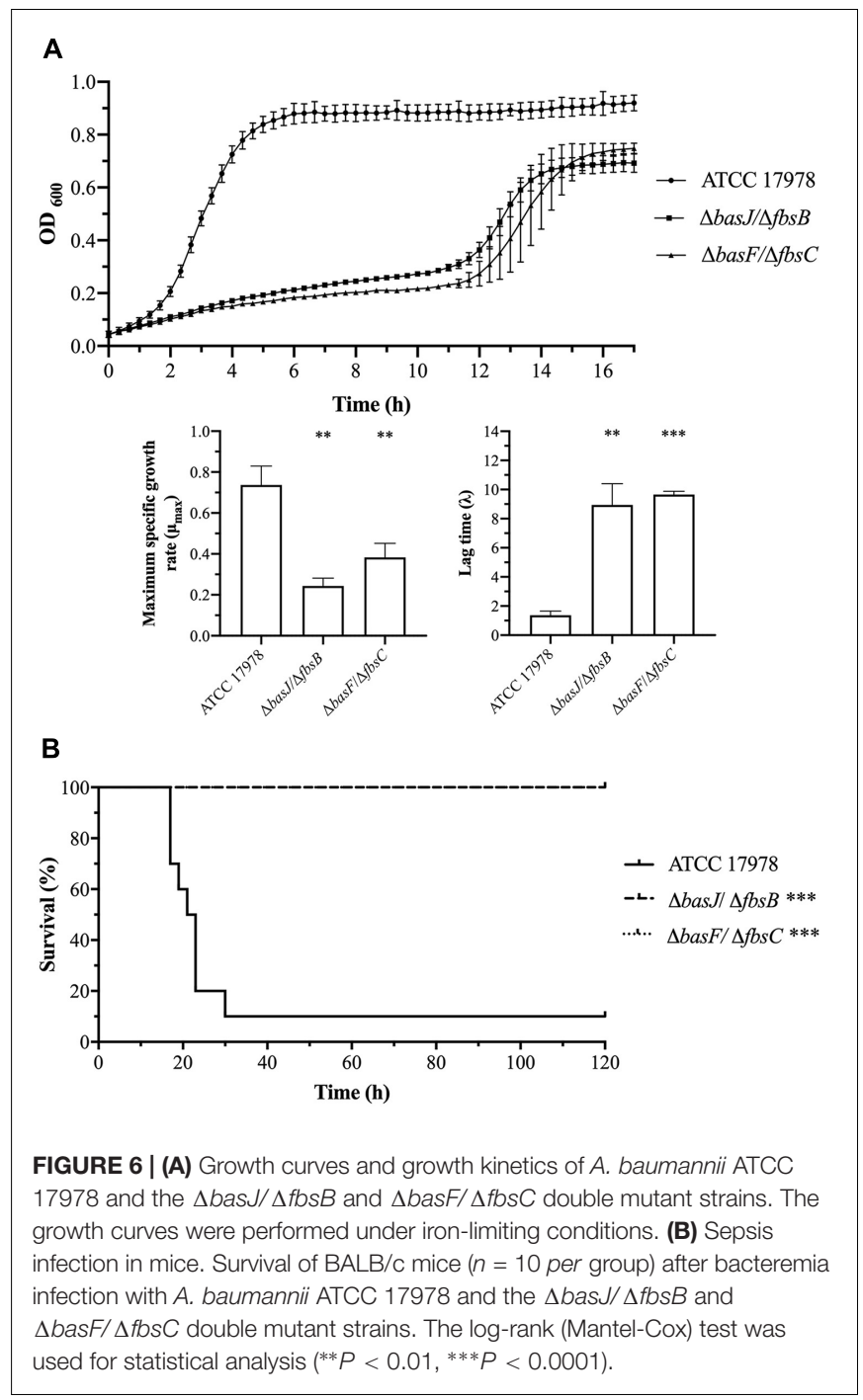

that the deletion of the entA gene will inhibit the biosynthesis of both acinetobactin and fimsbactin siderophores.

Deletion of $b a s B$ and $b a s D$ also led to a significant decrease in virulence characterized by an impaired fitness under ironlimiting conditions and increased mice survival. We have previously reported that $b a s B$ is an essential gene for the virulence of $A$. baumannii during pneumonia in mice and for bacteria growth under iron-limiting conditions (Martínez-Guitián et al., 2020). In addition, Gaddy et al. demonstrated that basD gene is essential for the biosynthesis of acinetobactin and for the bacterial growth under iron-depleted conditions in A. baumannii ATCC 19606 (Gaddy et al., 2012). Both genes code for proteins involved in the last steps of the biosynthetic pathway of acinetobactin, where DHBA and L-Threonine precursors are linked (BasD) and the resulting intermediate is bonded to $N$-hydroxyhistamine (BasB) to give preacinetobactin (Hasan et al., 2015; Shapiro and Wencewicz, 2016; Song and Kim, 2020). Both $\Delta$ basB and $\Delta$ basD mutants were unable to synthetize acinetobactin.

A closer analysis of the basJ gene, showed that its deletion did not have any effect in the biosynthesis of acinetobactin.
However, the deletion of both acinetobactin (basJ) and fimsbactin $(f b s B)$ redundant genes resulted in the loss of acinetobactin and fimsbactins production. This fact demonstrates that in absence of the basJ gene, $A$. baumannii ATCC 17978 can use $f b s B$ to synthesize acinetobactin. This is a clear example of molecular redundancy whereby two genes have the same function or when an alternative pathway fulfills the mission role of an inactivated gene. Pathogens used it to adapt to a continuous changing environment, avoiding the antimicrobial defenses of their hosts (Ghosh and O'Connor, 2017). Based on our results, we predict that the redundancy of $\triangle$ basF, $\Delta$ basI, $\triangle$ bas $H, \triangle$ bas $A$, and $\triangle$ basE strains possibly lead to the unchanged ability to synthesize acinetobactin.

Among the genes involved in the transport of acinetobactin, only the gen bauA, coding for the outer-membrane receptor, was found to be essential for the virulence during a murine sepsis model. Previous studies have shown that the gene bauA is essential for the virulence of $A$. baumannii ATCC 19606 since its deletion led to a decrease in the ability of the bacteria to infect, divide inside body fluids of mice and in fitness under iron-limiting conditions (Gaddy et al., 2012). In fact, BauA was proposed as a good vaccine candidate since mice injected with recombinant BauA were able to produce antibodies against this protein. In addition, passive immunization using serum antiBauA protected mice from infection (Esmaeilkhani et al., 2016). Our data slightly differ with this study since we have not observed any reduction in the fitness of $A$. baumannii ATCC 17978 when bauA was deleted. This discrepancy could be explained by the higher susceptibility of the strain ATCC 19606 to chelate iron (III) compared to the ATCC 17978 strain, possibly due to the lack of fimsbactins and baumanoferrin production in the ATCC 19606 strain (Antunes et al., 2011; Proschak et al., 2013; Penwell et al., 2015; Ramirez et al., 2019).

Both BarA and BarB proteins belong to the acinetobactin secretion system (efflux) of the ABC superfamily. Mutant strains lacking the genes involved in the synthesis of these proteins, $\triangle b a r A$ and $\triangle b a r B$, showed a significant decrease in fitness under iron-limiting conditions. Notwithstanding, no statistical differences between the percentage of survival of the mice infected with these mutant strains and the mice infected with the parental strain were observed. SPE-HLB/HPLC-MS analysis of $\triangle$ barB mutant strain cultures showed the presence of acinetobactin in its cell-free supernatant, which indicates that this single mutant strain did not prevent the efflux of acinetobactin outside the bacteria. A previous study carried out by Penwell et al. in A. baumannii ATCC 19606, showed a growth defect under iron-limiting conditions and a $60 \%$ decrease of acinetobactin effluxed in the $\triangle$ bar $A / \Delta$ barB cell-free supernatant compared with the parental strain (Penwel, 2013). The reduction in the efflux of acinetobactin matches with the partial loss of virulence in the $\triangle b a r A$ and $\triangle b a r B$ mutant strains. It is known that $A$. baumannii possesses a wide variety of transport mechanisms. It is possible that under stress conditions, the bacteria could use non-specific transporter systems to secrete and uptake acinetobactin and do not lose the iron-battle against the host (Iacono et al., 2008; Coyne et al., 2010, 2011; Fernando and Kumar, 2012). 
Several researchers have focused their efforts on the development of new inhibitors based on acinetobactin metabolism. Inhibitors of BasE, an enzyme involved in biosynthesis of the acinetobactin, have shown a powerful inhibitory activity (Neres et al., 2013). Analogous of acinetobactin have also shown bacteriostatic activity as they were able to block the transport of the iron-acinetobactin complex inside the bacteria (Bohac et al., 2017; Shapiro and Wencewicz, 2017). In the last years, siderophore conjugates using the "Trojan Horse" antibiotic drug delivery strategy has become more popular for combating this microorganism. In fact, cefiderocol (fetcroja) was the first cathecol-substituted siderophore cephalosporin approved by the FDA and EMA (Ji et al., 2012; Wencewicz and Miller, 2013; Ghosh et al., 2017; Parsels et al., 2021).

In summary, we performed an in-depth analysis of the role of each individual gene of the acinetobactin metabolism in the virulence of A. baumannii ATCC 17978, allowing us to identify six potential targets for the design of new antimicrobials against this microorganism: five of them involved in its biosynthesis (entA, basG, basC, basD, and basB) and one related to its transport $(\operatorname{bau} A)$. Due to the similar function and potentially similar structure of the enzymes involved in the biosynthesis of acinetobactin and fimsbactin, inhibitors against the remaining biosynthetic steps could also have the potential to be effective by inactivating both redundant proteins.

\section{DATA AVAILABILITY STATEMENT}

The original contributions presented in the study are included in the article/Supplementary Material, further inquiries can be directed to the corresponding author.

\section{ETHICS STATEMENT}

The animal study was reviewed and approved by Hospital Universitario A Coruña, Spain, project code P102.

\section{AUTHOR CONTRIBUTIONS}

KC-P, SR-F, NT-T, and LÁ-F performed mutant construction. MM-G, JV-U and KC-P performed phenotypic experiments and animal models. LA performed the analysis of the siderophorecontent. AB, MP, CJ, and LÁ-F designed and supervised the experiments and wrote the manuscript. GB and JR revised the manuscript. All authors read and approved the final manuscript.

\section{REFERENCES}

Álvarez-Fraga, L., Pérez, A., Rumbo-Feal, S., Merino, M., Vallejo, J. A., Ohneck, E. J., et al. (2016). Analysis of the role of the LH92_11085 gene of a biofilm hyper-producing Acinetobacter baumannii strain on biofilm formation and attachment to eukaryotic cells. Virulence 7, 443-455. doi: 10.1080/21505594. 2016.1145335

Álvarez-Fraga, L., Vázquez-Ucha, J. C., Martínez-Guitián, M., Vallejo, J. A., Bou, G., Beceiro, A., et al. (2018). Pneumonia infection in mice reveals the involvement

\section{FUNDING}

This work was funded by Projects PI15/00860 awarded to GB and PI17/01482 to $\mathrm{AB}$ and MP, all within in the National Plan for Scientific Research, Development and Technological Innovation 2013-2016 and funded by the ISCIII - General Subdirection of Assessment and Promotion of the ResearchEuropean Regional Development Fund (FEDER) "A way of making Europe." The study was also funded by project IN607A 2016/22 (GAIN- Agencia Gallega de Innovación - Consellería de Economía, Emprego e Industria) awarded to GB. This work was also supported by Planes Nacionales de I + D + i 20082011/2013-2016 and Instituto de Salud Carlos III, Subdirección General de Redes y Centros de Investigación Cooperativa, Ministerio de Economía y Competitividad, Spanish Network for Research in Infectious Diseases (REIPI RD16/0016/006) cofinanced by European Development Regional Fund "A way to achieve Europe" and operative program Intelligent Growth 20142020. This work was also supported by Grant RTI2018-093634B-C22 (AEI/FEDER, EU) from the State Agency for Research (AEI) of Spain, co-funded by the FEDER Programme from the European Union and Xunta de Galicia for the support of Grant ED431E 2018/03 for CICA-INIBIC strategic and the initiative "Seed Projects 2019-2020." JV-U was financially supported by the ISCIII project FI18/00315, LÁ-F by the ISCIII project PI14/00059 and the IN606B-2018/011, MM-G was financially supported by the Grant Clara Roy (SEIMC, Spanish Society of Clinical Microbiology and Infectious Diseases), KC-P by IN607A 2016/22 and AECC (Asociación Española Contra el Cáncer) predoctoral fellowship and LA by Xunta de Galicia co-funded with the European Social Fund (FSE) of the European Union (ED481A2019/081).

\section{ACKNOWLEDGMENTS}

We thank M. I. Voskuil (Dept. of Immunology and Microbiology, University of Colorado Medical School, CO, United States) for providing pMo130.

\section{SUPPLEMENTARY MATERIAL}

The Supplementary Material for this article can be found online at: https://www.frontiersin.org/articles/10.3389/fmicb. 2021.752070/full\#supplementary-material

of the feoA gene in the pathogenesis of Acinetobacter baumannii. Virulence 9, 496-509. doi: 10.1080/21505594.2017.1420451

Antunes, L. C., Imperi, F., Towner, K. J., and Visca, P. (2011). Genome-assisted identification of putative iron-utilization genes in Acinetobacter baumannii and their distribution among a genotypically diverse collection of clinical isolates. Res. Microbiol. 162, 279-284. doi: 10.1016/j.resmic.2010.10.010

Balado, M., Souto, A., Vences, A., Careaga, V. P., Valderrama, K., Segade, Y., et al. (2015). Two Catechol Siderophores, Acinetobactin and Amonabactin, Are Simultaneously Produced by Aeromonas salmonicida subsp. salmonicida 
Sharing Part of the Biosynthetic Pathway. ACS Chem. Biol. 10, 2850-2860. doi: 10.1021/acschembio.5b00624

Bohac, T. J., Shapiro, J. A., and Wencewicz, T. A. (2017). Rigid oxazole acinetobactin analog blocks siderophore cycling in Acinetobacter baumannii. ACS Infect. Dis. 3, 802-806. doi: 10.1021/acsinfecdis.7b0 0146

Coyne, S., Courvalin, P., and Périchon, B. (2011). Efflux-mediated antibiotic resistance in Acinetobacter spp. Antimicrob. Agents Chemother. 55, 947-953. doi: 10.1128/AAC.01388-10

Coyne, S., Rosenfeld, N., Lambert, T., Courvalin, P., and Périchon, B. (2010). Overexpression of resistance-nodulation-cell division pump AdeFGH confers multidrug resistance in Acinetobacter baumannii. Antimicrob. Agents Chemother. 54, 4389-4393. doi: 10.1128/AAC.00155-10

De Oliveira, D. M. P., Forde, B. M., Kidd, T. J., Harris, P. N. A., Schembri, M. A., Beatson, S. A., et al. (2020). Antimicrobial resistance in ESKAPE pathogens. Clin. Microbiol. Rev. 33:e00181-19. doi: 10.1128/CMR.00 181-19

Dickey, S. W., Cheung, G. Y. C., and Otto, M. (2017). Different drugs for bad bugs: antivirulence strategies in the age of antibiotic resistance. Nat. Rev. Drug Discov. 16, 457-471. doi: 10.1038/nrd.2017.23

Dorsey, C. W., Tomaras, A. P., Connerly, P. L., Tolmasky, M. E., Crosa, J. H., and Actis, L. A. (2004). The siderophore-mediated iron acquisition systems of Acinetobacter baumannii ATCC 19606 and Vibrio anguillarum 775 are structurally and functionally related. Microbiology 150, 3657-3667. doi: 10 . 1099/mic.0.27371-0

Eijkelkamp, B. A., Hassan, K. A., Paulsen, I. T., and Brown, M. H. (2011). Investigation of the human pathogen Acinetobacter baumannii under iron limiting conditions. BMC Genomics 12:126. doi: 10.1186/1471-216412-126

Esmaeilkhani, H., Rasooli, I., Nazarian, S., and Sefid, F. (2016). In vivo validation of the immunogenicity of recombinant Baumannii Acinetobactin Utilization A protein (rBauA). Microb. Pathog. 98, 77-81. doi: 10.1016/j.micpath.2016. 06.032

Espada, A., Anta, C., Bragado, A., Rodríguez, J., and Jiménez, C. (2011). An approach to speed up the isolation of hydrophilic metabolites from natural sources at semipreparative level by using a hydrophilic-lipophilic balance/mixed-mode strong cation exchange-high-performance liquid chromatography/mass spectrometry system. J. Chromatogr. A 1218, 1790-1794. doi: 10.1016/j.chroma.2011.01.072

Fernando, D., and Kumar, A. (2012). Growth phase-dependent expression of RND efflux pump- and outer membrane porin-encoding genes in Acinetobacter baumannii ATCC 19606. J. Antimicrob. Chemother. 67, 569-572. doi: 10.1093/ $\mathrm{jac} / \mathrm{dkr} 519$

Gaddy, J. A., Arivett, B. A., Mcconnell, M. J., López-Rojas, R., Pachón, J., and Actis, L. A. (2012). Role of acinetobactin-mediated iron acquisition functions in the interaction of Acinetobacter baumannii strain ATCC 19606T with human lung epithelial cells, Galleria mellonella caterpillars, and mice. Infect. Immun. 80, 1015-1024. doi: 10.1128/IAI.06279-11

Ghosh, M., Miller, P. A., Möllmann, U., Claypool, W. D., Schroeder, V. A., Wolter, W. R., et al. (2017). Targeted antibiotic delivery: selective siderophore conjugation with daptomycin confers potent activity against multidrug resistant Acinetobacter baumannii both in vitro and in vivo. J. Med. Chem. 60, 4577-4583. doi: 10.1021/acs.jmedchem.7b00102

Ghosh, S., and O'Connor, T. J. (2017). Beyond Paralogs: the multiple layers of redundancy in bacterial pathogenesis. Front. Cell Infect. Microbiol. 7:467. doi: 10.3389/fcimb.2017.00467

Hamad, M. A., Zajdowicz, S. L., Holmes, R. K., and Voskuil, M. I. (2009). An allelic exchange system for compliant genetic manipulation of the select agents Burkholderia pseudomallei and Burkholderia mallei. Gene 430, 123-131. doi: 10.1016/j.gene.2008.10.011

Harding, C. M., Hennon, S. W., and Feldman, M. F. (2018). Uncovering the mechanisms of Acinetobacter baumannii virulence. Nat. Rev. Microbiol. 16, 91-102. doi: 10.1038/nrmicro.2017.148

Hasan, T., Choi, C. H., and Oh, M. H. (2015). Genes involved in the biosynthesis and transport of Acinetobactin in Acinetobacter baumannii. Genomics Inform. 13, 2-6. doi: 10.5808/GI.2015.13.1.2

Iacono, M., Villa, L., Fortini, D., Bordoni, R., Imperi, F., Bonnal, R. J., et al. (2008). Whole-genome pyrosequencing of an epidemic multidrug-resistant
Acinetobacter baumannii strain belonging to the European clone II group. Antimicrob. Agents Chemother. 52, 2616-2625. doi: 10.1128/AAC.01643-07

Ji, C., Juárez-Hernández, R. E., and Miller, M. J. (2012). Exploiting bacterial iron acquisition: siderophore conjugates. Future Med. Chem. 4, 297-313. doi: 10. 4155/fmc.11.191

Martínez-Guitián, M., Vázquez-Ucha, J. C., Álvarez-Fraga, L., Conde-Pérez, K., Lasarte-Monterrubio, C., Vallejo, J. A., et al. (2019). Involvement of HisF in the Persistence of Acinetobacter baumannii during a pneumonia infection. Front. Cell. Infect. Microbiol. 9:310. doi: 10.3389/fcimb.2019.00310

Martínez-Guitián, M., Vázquez-Ucha, J. C., Álvarez-Fraga, L., Conde-Pérez, K., Vallejo, J. A., Perina, A., et al. (2020). Global transcriptomic analysis during murine pneumonia infection unravels new virulence factors in Acinetobacter baumannii. J. Infect. Dis. 223, 1356-1366. doi: 10.1093/infdis/jia a522

Mihara, K., Tanabe, T., Yamakawa, Y., Funahashi, T., Nakao, H., Narimatsu, S., et al. (2004). Identification and transcriptional organization of a gene cluster involved in biosynthesis and transport of acinetobactin, a siderophore produced by Acinetobacter baumannii ATCC 19606T. Microbiology 150, 2587-2597. doi: 10.1099/mic.0.27141-0

Moynié, L., Serra, I., Scorciapino, M. A., Oueis, E., Page, M. G., Ceccarelli, M., et al. (2018). Preacinetobactin not acinetobactin is essential for iron uptake by the BauA transporter of the pathogen Acinetobacter baumannii. eLife 7:e42270. doi: 10.7554/eLife.42270.027

Murray, G. L., Tsyganov, K., Kostoulias, X. P., Bulach, D. M., Powell, D., Creek, D. J., et al. (2017). Global gene expression profile of Acinetobacter baumannii during bacteremia. J. Infect. Dis. 215, S52-S57. doi: 10.1093/infdis/ jiw529

Neres, J., Engelhart, C. A., Drake, E. J., Wilson, D. J., Fu, P., Boshoff, H. I., et al. (2013). Non-nucleoside inhibitors of BasE, an adenylating enzyme in the siderophore biosynthetic pathway of the opportunistic pathogen Acinetobacter baumannii. J. Med. Chem. 56, 2385-2405. doi: 10.1021/jm301 709s

Parsels, K. A., Mastro, K. A., Steele, J. M., Thomas, S. J., and Kufel, W. D. (2021). Cefiderocol: a novel siderophore cephalosporin for multidrug-resistant Gramnegative bacterial infections. J. Antimicrob. Chemother. 76, 1379-1391. doi: 10.1093/jac/dkab015

Penwel, W. F. (2013). Iron Acquisition in Acinetobacter baumannii. Ph.D. thesis. Oxford: Miami University.

Penwell, W. F., Arivett, B. A., and Actis, L. A. (2012). The Acinetobacter baumannii entA gene located outside the acinetobactin cluster is critical for siderophore production, iron acquisition and virulence. PLoS One 7:e36493. doi: 10.1371/ journal.pone.0036493

Penwell, W. F., Degrace, N., Tentarelli, S., Gauthier, L., Gilbert, C. M., Arivett, B. A., et al. (2015). Discovery and Characterization of New Hydroxamate Siderophores, Baumannoferrin A and B, produced by Acinetobacter baumannii. Chembiochem 16, 1896-1904. doi: 10.1002/cbic.201500147

Proschak, A., Lubuta, P., Grün, P., Löhr, F., Wilharm, G., De Berardinis, V., et al. (2013). Structure and biosynthesis of fimsbactins A-F, siderophores from Acinetobacter baumannii and Acinetobacter baylyi. Chembiochem 14, 633-638. doi: 10.1002/cbic. 201200764

Ramirez, M. S., Penwell, W. F., Traglia, G. M., Zimbler, D. L., Gaddy, J. A., Nikolaidis, N., et al. (2019). Identification of potential virulence factors in the model strain Acinetobacter baumannii A118. Front. Microbiol. 10:1599. doi: $10.3389 /$ fmicb.2019.01599

Shapiro, J. A., and Wencewicz, T. A. (2016). Acinetobactin isomerization enables adaptive iron acquisition in Acinetobacter baumannii through $\mathrm{pH}$-triggered siderophore swapping. ACS Infect. Dis. 2, 157-168. doi: 10.1021/acsinfecdis. 5 b00145

Shapiro, J. A., and Wencewicz, T. A. (2017). Structure-function studies of acinetobactin analogs. Metallomics 9, 463-470. doi: 10.1039/C7MT00 064B

Sheldon, J. R., Laakso, H. A., and Heinrichs, D. E. (2016). Iron acquisition strategies of bacterial pathogens. Microbiol. Spectr. 4:2. doi: 10.1128/97815558192 86.ch3

Sheldon, J. R., and Skaar, E. P. (2020). Acinetobacter baumannii can use multiple siderophores for iron acquisition, but only acinetobactin is required for virulence. PLoS Pathog. 16:e1008995. doi: 10.1371/journal.ppat.100 8995 
Song, W. Y., and Kim, H. J. (2020). Current biochemical understanding regarding the metabolism of acinetobactin, the major siderophore of the human pathogen Acinetobacter baumannii, and outlook for discovery of novel anti-infectious agents based thereon. Nat. Prod. Rep. 37, 477-487. doi: 10.1039/C9NP00 $046 \mathrm{~A}$

Tjørve, K. M. C., and Tjørve, E. (2017). The use of Gompertz models in growth analyses, and new Gompertz-model approach: an addition to the UnifiedRichards family. PLoS One 12:e0178691. doi: 10.1371/journal.pone.0178691

Wencewicz, T. A., and Miller, M. J. (2013). Biscatecholate-monohydroxamate mixed ligand siderophore-carbacephalosporin conjugates are selective sideromycin antibiotics that target Acinetobacter baumannii. J. Med. Chem. 56, 4044-4052. doi: 10.1021/jm400265k

Wong, D., Nielsen, T. B., Bonomo, R. A., Pantapalangkoor, P., Luna, B., and Spellberg, B. (2017). Clinical and pathophysiological overview of acinetobacter infections: a century of challenges. Clin. Microbiol. Rev. 30, 409-447. doi: 10.1128/CMR.00058-16

World Health Organization [WHO] (2017). Global Priority List of AntibioticResistant Bacteria to Guide Research, Discovery, and Development of New Antibiotics. Available online at: http://www.who.int/medicines/publications/ WHO-PPL-Short_Summary_25Feb-ET_NM_WHO.pdf?ua=1 (accessed June $25,2021)$.

Yamamoto, S., Okujo, N., and Sakakibara, Y. (1994). Isolation and structure elucidation of acinetobactin, a novel siderophore from Acinetobacter baumannii. Arch. Microbiol. 162, 249-254. doi: 10.1007/BF00301846
Conflict of Interest: The authors declare that the research was conducted in the absence of any commercial or financial relationships that could be construed as a potential conflict of interest.

Publisher's Note: All claims expressed in this article are solely those of the authors and do not necessarily represent those of their affiliated organizations, or those of the publisher, the editors and the reviewers. Any product that may be evaluated in this article, or claim that may be made by its manufacturer, is not guaranteed or endorsed by the publisher.

Citation: Conde-Pérez K, Vázquez-Ucha JC, Álvarez-Fraga L, Ageitos L, RumboFeal S, Martínez-Guitián M, Trigo-Tasende N, Rodríguez J, Bou G, Jiménez C, Beceiro A and Poza M (2021) In-Depth Analysis of the Role of the Acinetobactin Cluster in the Virulence of Acinetobacter baumannii. Front. Microbiol. 12:752070. doi: 10.3389/fmicb.2021.752070

Copyright (c) 2021 Conde-Pérez, Vázquez-Ucha, Álvarez-Fraga, Ageitos, RumboFeal, Martínez-Guitián, Trigo-Tasende, Rodríguez, Bou, Jiménez, Beceiro and Poza. This is an open-access article distributed under the terms of the Creative Commons Attribution License (CC BY). The use, distribution or reproduction in other forums is permitted, provided the original author(s) and the copyright owner(s) are credited and that the original publication in this journal is cited, in accordance with accepted academic practice. No use, distribution or reproduction is permitted which does not comply with these terms. 